\title{
Modelling Cracked Cross-Ply Laminates with Delamination Buckling
}

\author{
A. Köllner ${ }^{1, a}$, M. Kashtalyan ${ }^{2, b}$, I Guz ${ }^{2}$, C. Völlmecke ${ }^{1}$ \\ ${ }^{1}$ Technische Universität Berlin, Institute of Mechanics, Stability and failure of functionally optimized \\ structures Group, Einsteinufer 5, 10587 Berlin \\ ${ }^{2}$ University of Aberdeen, School of Engineering, Centre for Micro- and Nanomechanics, Fraser \\ Noble Building, Aberdeen AB24 3UE, Scotland, United Kingdom \\ aanton.koellner@tu-berlin.de, bm.kashtalyan@abdn.ac.uk,
}

Keywords: Matrix cracks, Delamination, Delamination Buckling, Composites, Plates.

\begin{abstract}
The mechanical behavior of cross-ply laminates loaded under in-plane compression containing matrix cracks and delaminations is investigated in order to study their influence on the structural stability behavior. This is done by employing a semi-analytical modelling approach which comprises an analytical framework for a structural stability analysis of damageable structures and the Equivalent Constrained Model for deriving reduced stiffness properties of the cracked layers. Cross-ply laminates with varying delamination depths as well as varying matrix crack densities are studied.
\end{abstract}

\section{Introduction}

The current work aims at studying the effect of material damage on the structural stability behavior of composite laminates. Two well-known types of material damage are delaminations and matrix cracks. Whereas the influence of delaminations on the compressive behavior of composite panels is well-investigated (e.g. [1-3]), the presence of matrix cracks is omitted when studying delamination buckling. Furthermore, until recently, the structural stability behavior once delaminations growth is caused has been solely modelled using finite element formulations (e.g. [4]). With the analytical framework described in [5], damage growth, e.g. delamination growth, can be considered in a structural stability analysis.

The present study is an extension of the work in [1,5] investigating delaminated composite laminates, so that the presence of matrix cracks is accounted for in the semi-analytical modelling approach. The cracked layer is modelled as an equivalent homogeneous layer with degraded stiffness properties, which are determined in a closed form with the aid of the Equivalent Constraint Model [6, 7].

\section{Semi-Analytical Model}

Geometric model. The geometric model of a delaminated composite strut is shown in Fig. 1. The strut is subdivided into four parts indicated by the circled numerals. Parts 1 and 2 describe the upper and lower sublaminate respectively. Parts 3 and 4 are assigned to the undelaminated region. The delamination is assumed to be centered and its depth is defined by the parameter $a$. Matrix cracks are assumed to be equally distributed over the entire length of a layer indicated by the grey area. Matrix cracks are only considered in the off-axis $\left(90^{\circ}\right)$ layers of the cross-play laminates to be studied. Five generalized coordinates are employed to describe the deformation behavior of the strut.

The generalized coordinates $q_{1}$ and $q_{2}$ are the amplitudes of the upper and lower sublaminate respectively. The rotation at the interface between the delaminated and undelaminated region is described by $q_{3}$. The axial shortening of the delaminated and undelaminated region constitutes the generalized coordinates $q_{4}$ and $q_{5}$ respectively (not shown in Fig. 1). A single loading parameter in the form of a uniaxial compressive force $P$ is applied to the strut. 


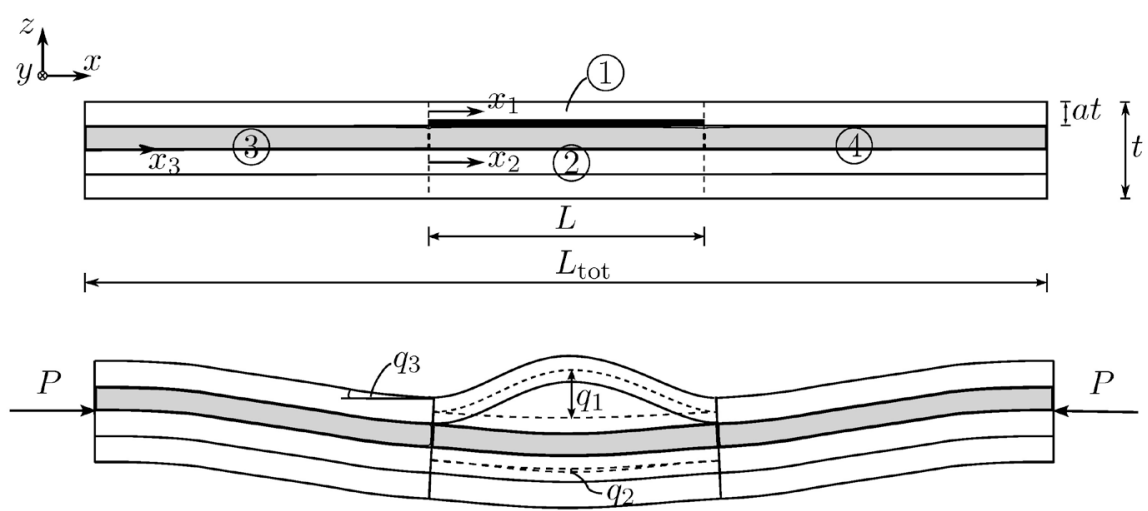

Fig. 1: Geometric model of a delaminated multi-layered laminate with matrix cracks.

Two damage parameters are considered in the geometric model. The delamination length is denoted by $L$ and a relative matrix crack density, $D$, given by the ratio of the thickness of a single layer to the spacing in between two matrix cracks is employed.

Structural stability formalism. The modelling approach strongly follows the analytical framework presented in [5]. The deformation process is strictly separated into a conservative part, i.e. the damage parameters remain constant, and a non-conservative part, i.e. damage growth occurs from one loading steps to another. In the current work, focus is placed on investigating the influence of given matrix crack densities on the phenomenon delamination growth during delamination buckling. Thus, only the delamination is considered for damage growth.

A RAYLEIGH-RITZ formulation is employed. The approximation of the buckling displacement and the axial shortening is taken from $[1,5]$. Thus, the conservative part of the deformation process is modelled using the well-known total potential energy principle, i.e. $\delta \Pi\left(q_{i}\right)=0$, where $\Pi$ comprises the strain energy and the work done by the applied force, yielding the deformation path associated with non-growing damage $q_{i}(P)$.

The deformation state causing delamination growth is determined by calculating the energy release rate, $G=-\partial \Pi /(b \partial L)$, using the deformation path $q_{i}(P)$ where $b$ is the width of the strut. Whenever the energy release rate reaches the critical energy release rate $G_{\mathrm{c}}$, i.e. $G \geq G_{\mathrm{c}}$, delamination growth occurs. The deformation state at which growth is initiated is denoted by $S_{0}=\left(q_{i}^{0}, P^{0}\right)$ and is referred to as damage state.

The formalism employs that during stable delamination growth equality holds in between the energy release rate and the critical energy release rate [5]. Thus, the delamination length is implicitly given by rewriting the equality $G=G_{\mathrm{c}}$ and assuming that a unique solution exists, i.e.

$$
G-G_{\mathrm{c}}=D\left(q_{i}, P, L\left(q_{i}, P\right)\right) \equiv 0 .
$$

As described in [5], an explicit form of the delamination length can be derived by employing a TAYLOR series approximation around the damage state $S_{0}$, where the partial derivatives required are obtained from Eq. (1), thus

$$
\begin{aligned}
L\left(q_{i}, P\right)= & L^{0}+\left.\frac{\partial L}{\partial q_{i}}\right|_{S_{0}}\left(q_{i}-q_{i}^{0}\right)+\left.\frac{\partial L}{\partial P}\right|_{S_{0}}\left(P-P^{0}\right)+\left.\frac{1}{2} \frac{\partial^{2} L}{\partial q_{i} \partial q_{j}}\right|_{S_{0}}\left(q_{i}-q_{i}^{0}\right)\left(q_{j}-q_{j}^{0}\right)+ \\
& \left.\frac{\partial^{2} L}{\partial q_{i} \partial P}\right|_{S_{0}}\left(q_{i}-q_{i}^{0}\right)\left(P-P^{0}\right)+\left.\frac{1}{2} \frac{\partial L}{\partial P^{2}}\right|_{S_{0}}\left(P-P^{0}\right)^{2}+O(3),
\end{aligned}
$$

with $L^{0}$ being the initial pre-existing delamination length.

With the delamination length expressed in terms of the generalized coordinates and the compressive force (cf. Eq. (2)), a new governing functional can be derived, viz. an extended total 
potential energy $\Pi^{*}$ which comprises the total potential energy and the dissipative energy associated with delamination growth, $W_{\mathrm{D}}$, where the delamination length is replaced by the functions obtained, i.e.

$$
\Pi^{*}=\Pi\left(q_{i}, P, L\left(q_{i}, P\right)\right)+W_{\mathrm{D}}\left(L\left(q_{i}, P\right)\right) .
$$

The deformation path $q_{i}(P)$ starting from the deformation state causing delamination growth is obtained by the variational principle

$$
\delta \Pi^{*}\left(q_{i}\right)=0 .
$$

Inserting the solution from Eq. (4) into the functions obtained by the TAYLOR series (Eq. (2)) yields the behavior of the delamination length.

Effective stiffness of the cracked layer. To determine the effective stiffness properties of the cracked $90^{\circ}$ layer, an equivalent $\left[0^{\circ} / 90^{\circ}\right]_{\mathrm{s}}$ cross-ply laminate is considered with a ply thickness equal to half ply thickness of the original laminate. The reduced stiffness matrix of the equivalent homogeneous layer is given by

$$
\left[Q^{(90)}\right]=\left[\hat{Q}^{(90)}\right]-\left[\begin{array}{ccc}
\frac{\left(\hat{Q}_{12}^{(90)}\right)^{2}}{\hat{Q}_{22}^{(90)}} \Lambda_{22}^{(90)} & \hat{Q}_{12}^{(90)} \Lambda_{22}^{(90)} & 0 \\
\hat{Q}_{12}^{(90)} \Lambda_{22}^{(90)} & \hat{Q}_{22}^{(90)} \Lambda_{22}^{(90)} & 0 \\
0 & 0 & \hat{Q}_{66}^{(90)} \Lambda_{66}^{(90)}
\end{array}\right]
$$

where $\left[\hat{Q}^{(90)}\right]$ is the stiffness matrix of the undamaged layer, $\Lambda_{j j}^{(90)}=\Lambda_{j j}^{(90)}(D), j=2,6$, are the Insitu Damage Effective Functions (IDEFs), and $D$ is the relative matrix crack density. Closed-form expressions for IDEFs are derived from the stress analysis of the equivalent laminate as

$$
\Lambda_{j j}^{(90)}=1-\left[1-\frac{D}{\lambda_{i}^{(90)}} \tanh \frac{\lambda_{i}^{(90)}}{D}\right]\left[1-\alpha_{i}^{(90)} \frac{D}{\lambda_{i}^{(90)}} \tanh \frac{\lambda_{i}^{(90)}}{D}\right]^{-1}, j=2,6 ; \quad i=1,2 .
$$

Constants $\lambda_{i}^{(90)}$ and $\alpha_{i}^{(90)}, i=1,2$ depend solely on the elastic properties of undamaged $0^{\circ}$ and $90^{\circ}$ layers and the layer thickness ratio and are given in detail in $[6,7]$.

\section{Results}

Figs. 2 to 9 show the post-buckling responses in terms of normalized applied load against normalized midpoint deflections of the sublaminates (Figs. 2, 4, 6, 8) and normalized load against normalized end-shortening of the strut (Figs. 3, 5, 7, 9). The load and the end-shortening are normalized against the respective buckling load and shortening of an intact strut respectively. The midpoint deflection is normalized against the total thickness of the strut.

A cross-ply laminate $\left[0^{\circ} /\left(90^{\circ} / 0^{\circ}\right)_{7}\right]$ is investigated. Matrix cracks are assigned to the most outward $90^{\circ}$ layer in the laminate on the opposite side of the delamination. Three delamination depths $(a=\{1 / 15,2 / 15,3 / 15\}$, cf. Fig. 1$)$ and three matrix crack densities $(D=\{0.0,0.5,1.0\})$ are studied. A delamination length of $L_{\text {norm }}=L / L_{\text {tot }}=0.31$ and the conservative measure of $G_{\mathrm{c}}=G_{\mathrm{c}}^{\mathrm{I}}$ are considered. The dimensions of the strut and the material parameters are taken from $[1,5]$ and are listed in Table 1 . 
Table 1: Dimensions and material parameters used.

\begin{tabular}{cccc}
\hline & Dimensions & \multicolumn{2}{c}{ Material parameters } \\
\hline$L_{\text {tot }}$ & $96.52 \mathrm{~mm}$ & $E_{11}$ & $137.90 \mathrm{GPa}$ \\
$L$ & $30^{\circ} \mathrm{mm}$ & $E_{22}$ & $8.98 \mathrm{GPa}$ \\
$b$ & $12.7 \mathrm{~mm}$ & $G_{12}$ & $7.20 \mathrm{GPa}$ \\
$t$ & $1.1335 \mathrm{~mm}$ & $v_{12}$ & 0.30 \\
$a$ & $\{1 / 15,2 / 15,3 / 15\}$ & $G_{\mathrm{c}}^{\mathrm{I}}$ & $0.19 \mathrm{~N} / \mathrm{mm}$ \\
& & $D$ & $\{0.0,0.5,1.0\}$ \\
\hline
\end{tabular}

The post-buckling behavior omitting delamination growth and matrix cracks is illustrated in Figs. 2 and 3 for the case of $a=3 / 15$. In general, the response can be subdivided into a local buckling response, i.e. only the upper less stiff sublaminate buckles (cf. Fig.2 dashed line), and a global buckling response, i.e. the intact part also undergoes buckling. In the global buckling regime, the system almost loses its entire compressive stiffness as indicated in Fig. 3 which is characteristic for struts.

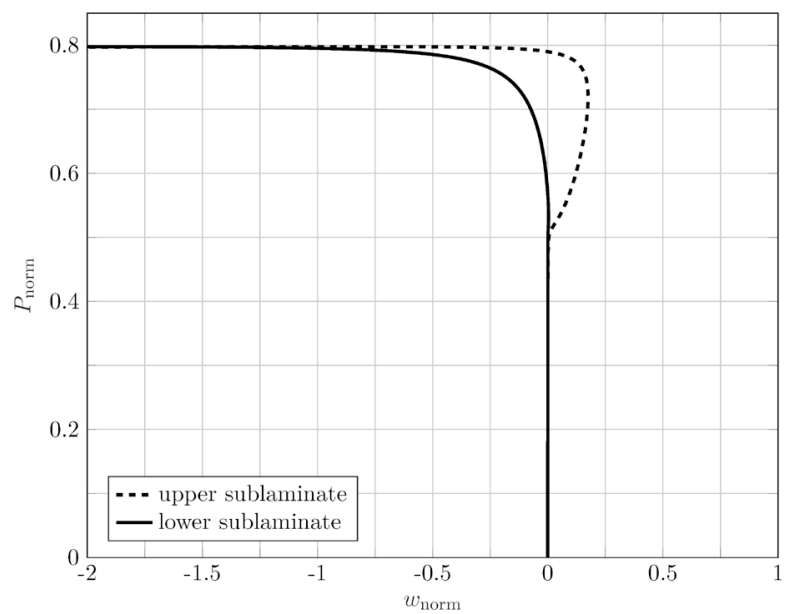

Fig. 2: $P_{\text {norm }}$ vs. $w_{\text {norm }}, a=3 / 15$, no growth.

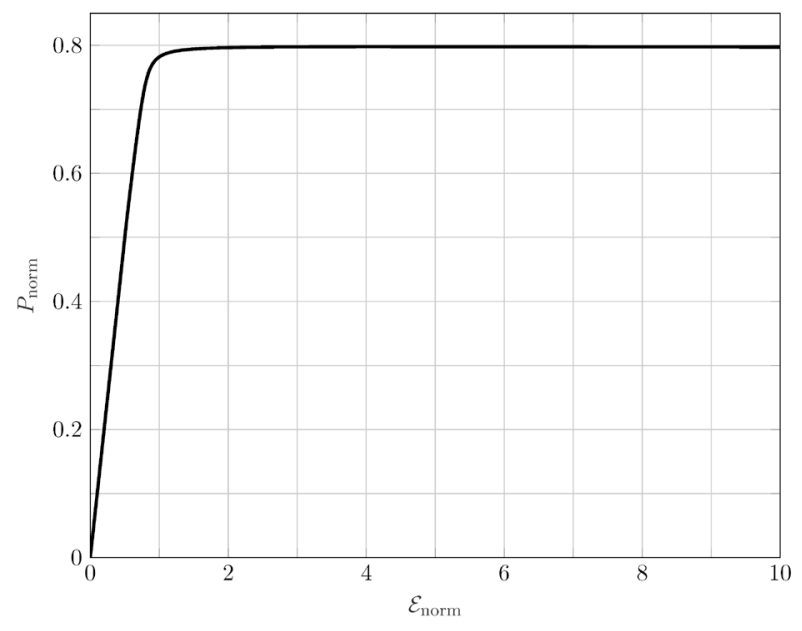

Fig. 3: $P_{\text {norm }}$ vs. $\varepsilon_{\text {norm }}, a=3 / 15$, no growth.

Besides providing general insight into the structural stability behavior of delaminated struts, Figs. 2 and 3 also serve for illustration purposes regarding the influence of delamination growth and matrix cracks on the post-buckling behavior, which is addressed next. All subsequent figures (Figs. 4 to 9) focus on the region associated with delamination growth.

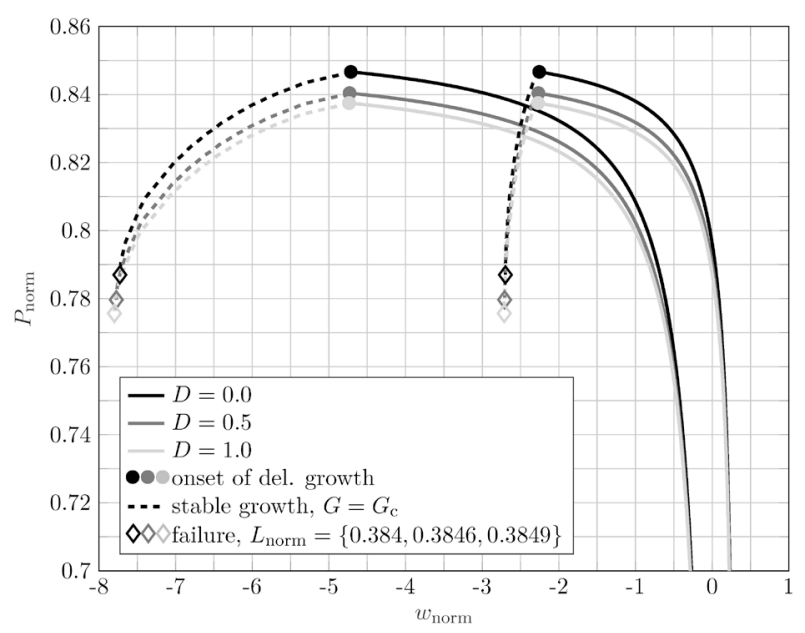

Fig. 4: $P_{\text {norm }}$ vs. $w_{\text {norm }}, a=1 / 15$.

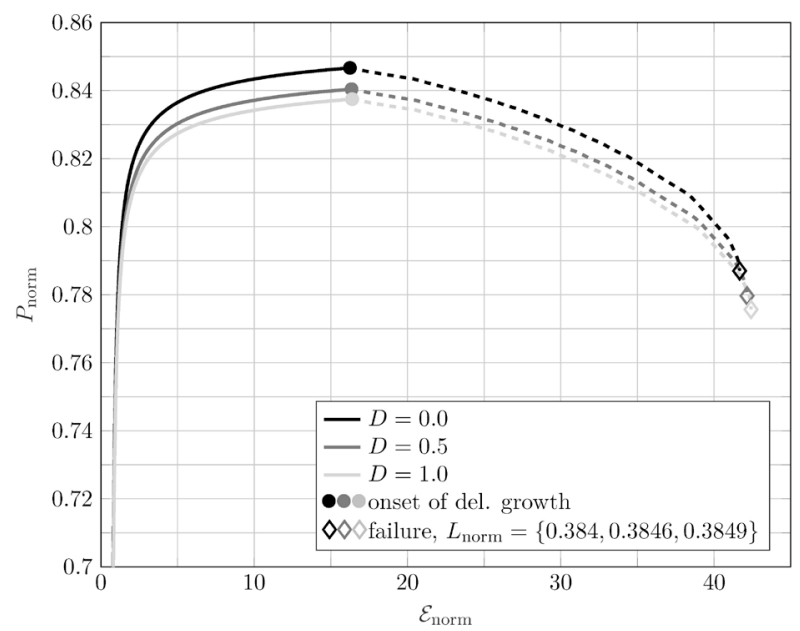

Fig. 5: $P_{\text {norm }}$ vs. $\varepsilon_{\text {norm }}, a=1 / 15$. 


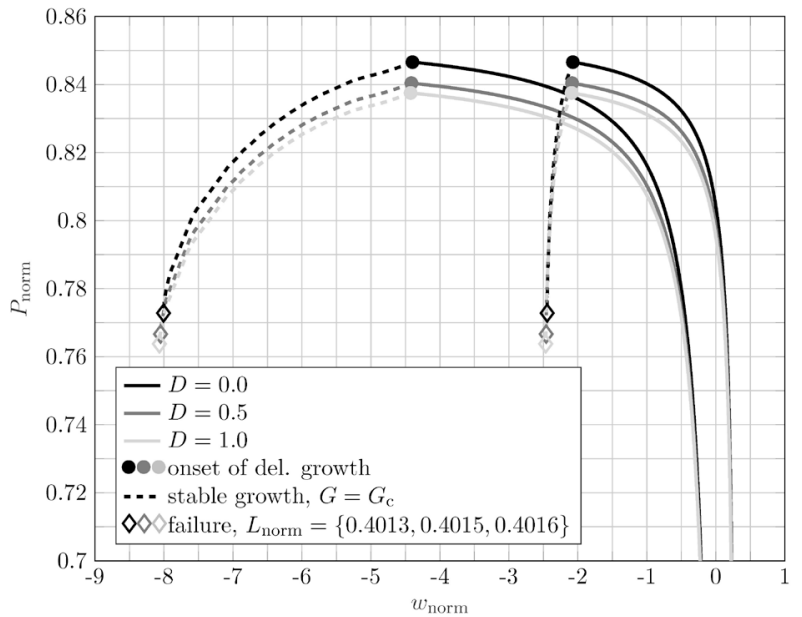

Fig. 6: $P_{\text {norm }}$ vs. $w_{\text {norm }}, a=2 / 15$.

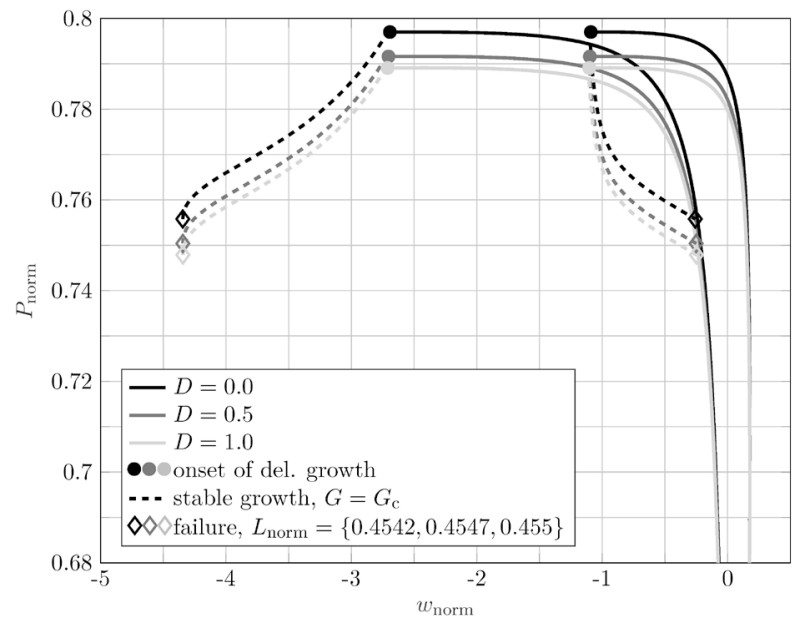

Fig. 8: $P_{\text {norm }}$ Vs. $w_{\text {norm }}, a=3 / 15$.

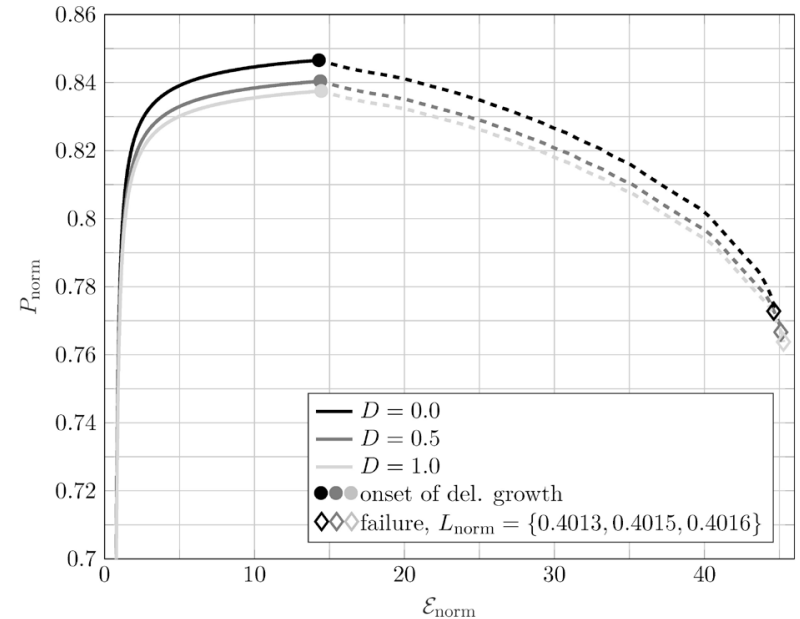

Fig. 7: $P_{\text {norm }}$ vs. $\varepsilon_{\text {norm }}, a=2 / 15$.

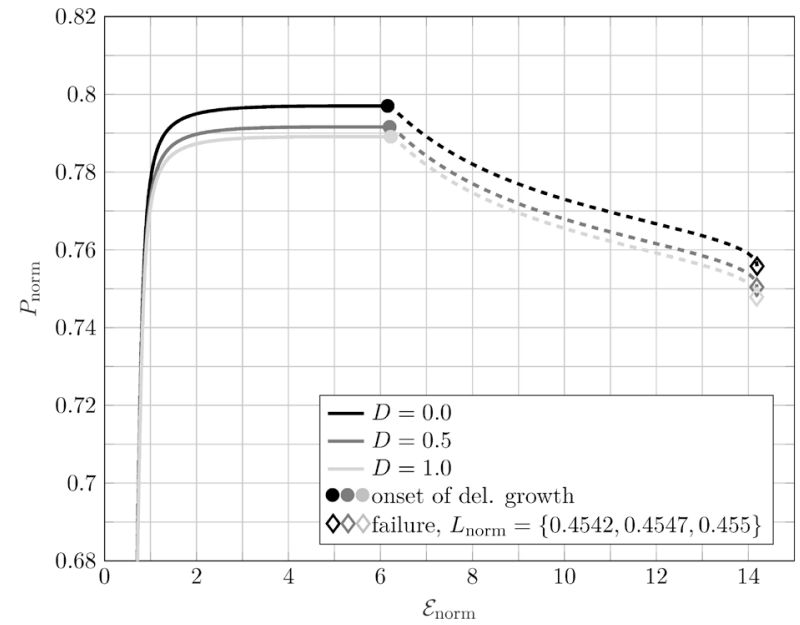

Fig. 9: $P_{\text {norm }}$ vs. $\varepsilon_{\text {norm }}, a=3 / 15$.

The deformation state at which delamination growth is generated is indicated in Figs. 4 to 9 by the symbol " $\bullet$ ". The figures provide the post-buckling response until the deformation state is reached which is associated with material failure (the symbol " $\diamond$ " in Figs. 4 to 9 ). Failure occurs whenever unstable delamination growth is caused yielding a sudden and complete separation of the strut. In contrast to Figs. 2 and 3, dashed lines in Figs. 4 to 9 indicate the part of the response which is associated with delamination growth.

The results provide the following insight:

- Delamination growth occurs during the global buckling response, i.e. once the intact part of the strut buckles. Significantly larger end-shortenings and loads are required to cause delamination growth for the cases $a=\{1 / 15,2 / 15\}$ than $a=3 / 15$.

- The qualitative post-buckling behavior remains unchanged with increasing matrix crack density. The loads causing delamination growth as well as failure of the system reduce by approximately $1-1.5 \%$, whereby the influence appears to slightly decrease with deeper delaminations.

- Increasing matrix crack densities cause marginally softer responses, thus growth occurs at slightly larger end-shortenings. Furthermore, the deformation state causing unstable delamination growth, i.e. failure of the system, is associated with larger delamination lengths. 


\section{Conclusions}

The study has shown that the presence of a single layer exhibiting matrix cracks does not affect the structural stability behavior of cross-ply laminates qualitatively, even though quantitatively the characteristic loads reduce and the end-shortenings as well as the delamination lengths at which failure occurs increase. Despite the minor effect, whenever configurations of struts are present where small changes in delamination length and depth cause a change in the buckling response (cf. $[1-3,5]$ ), the presence of matrix cracks may contribute towards the initiation of structural instability and material failure.

\section{Acknowledgements}

The authors gratefully acknowledge the support received by the German Research Foundation (DFG) under reference VO 2026/6-1.

\section{References}

[1] Köllner A, Jungnickel R, Völlmecke C. Delamination growth in buckled composite struts. Int J Fract (2016), 202(2), 261-269.

[2] Hunt GW, Hu B, Butler R, Almond DP, Wright JE. Nonlinear modeling of delaminated struts. AIAA Journal (2004), 42(11), 2364-2372.

[3] Köllner A, Völlmecke C. Buckling and postbuckling of delaminated composite struts. Int J Comput Meth Eng Sci Mech (2017), 18(1), 25-33.

[4] Nilsson K-F, Asp LE, Alpman JE, Nystedt L. Delamination buckling and growth for delaminations at different depths in a slender composite panel, Int J Solids Struct (2001), 38, 3039-3071.

[5] Köllner A, Völlmecke C. An analytical framework to extend the general structural stability analysis by considering certain inelastic effects - theory and application to delaminated composites. Compos Struct (2017), 170, 261-270.

[6] Kashtalyan M, Soutis C. Predicting residual stiffness of cracked composite laminates subjected to multi-axial in-plane loading. J Compos Mater (2013), 47(20-21), 2513-2524.

[7] Kashtalyan M, Soutis C. Modelling of stiffness degradation due to cracking in laminates subjected to multi-axial loading. Philos Trans Royal Soc A (2016), 374, 20160017. 